\title{
New aspects of cognition domains and psychopathological measures in psychiatry
}

\author{
Daniela Reich-Erkelenz • Andrea Schmitt • \\ Peter Falkai
}

Published online: 21 October 2014

(C) Springer-Verlag Berlin Heidelberg 2014

Irritable bowel syndrome (IBS) including its subtypes (diarrhea, constipation, mixed states) has regularly been associated with anxiety and depressive symptom levels. Fond et al. [1] can now confirm this assumption via a systematic review and meta-analysis after screening published studies and accessible theses within five wellestablished databases. Despite partly questionable study quality, the review of altogether 885 patients and 1,384 healthy controls in ten studies yielded significant evidence for higher anxiety and depression levels in patients compared with controls with inconsistencies for the IBS subtypes, which require deeper attentiveness. In their welldesigned study, Yen et al. [2] addressed the difficulties in cognitive control and working memory in 63 Chinese women suffering from premenstrual dysphoric disorder (PMDD) versus 74 controls via exploring effects of gonadotropic hormone and polymorphism of serotonin 1A receptor (HTR1A; rs6295-please pay attention to the authors' erratum [10] concerning false spellings in Tables 1, 2, and 4 plus Figure 1D-F) on cognitive deficit. Assessing working memory via the Go/Nogo, 2-back, and 3-back tasks for three menstrual cycles, the hypothesized cognitive decline in women with PMDD could be confirmed. The authors furthermore found an association of the G/G genotype of HTR1A (rs6295) with poor working

\section{Reich-Erkelenz}

Institute of Psychiatric Phenomics and Genomics, Ludwig-Maximilians-University Munich, Nußbaumstr. 7, 80336 Munich, Germany

\section{A. Schmitt $(\bowtie) \cdot$ P. Falkai}

Department of Psychiatry and Psychotherapy,

Ludwig-Maximilians-University Munich, Nußbaumstr. 7,

80336 Munich, Germany

e-mail: andrea.schmitt@med.uni-muenchen.de memory in the premenstrual phase which points to a reduced serotonin neurotransmission in PMDD. The Go/ Nogo task was also used by Ko et al. [3] in their fMRI study of Internet gaming disorder (IGD) focusing on impulsivity and brain correlates of response inhibition and error processing in 26 affected subjects compared with 23 controls. The authors found not only the expected higher impulsivity in IGD patients but also higher brain activation on response inhibition, especially over the left frontal lobe and bilateral caudate nucleus, while the activation over the right insula was lower compared with controls. These findings support the assumption of the frontostriatal plus salience network contributing to error processing.

Because the positive, negative, and general PANSS subscales do not exactly reflect the underlying dimensions, Woodward et al. [4] compared them to an own previously developed five-dimensional PANSS structure (positive symptoms, negative signs, disorganization, excitement, and emotional distress) in a sample of 610 patients with diagnosed schizophrenia, major depression, schizoaffective, or bipolar disorder admitted to a Canadian treatment refractory psychosis program in 11 years. The authors found confirmation of the hypothesized applicability of their PANSS structure which well fit the data at admission and discharge, showing positive symptoms to respond better to treatment than all other symptom dimensions albeit more pronounced in the schizoaffective and bipolar groups. The major depression group presented the best improvement in negative symptoms and emotional distress and the bipolar group, again, in disorganization. Other than schizoaffective disorder, schizophrenia showed reduced treatment response on all symptom dimensions. Thus, the proposed PANSS five-dimensional structure can inform clinicians about expectable symptom profiles at admission and discharge of refractory psychosis patients. 
Gorynia et al. [5] assumed an influence of eye dominance paired with cannabis use on intermanual coordination (IMC) and negative schizophrenia symptoms. They found eye dominance to affect IMC in 52 right- and 51 lefthanded in-patients with either eye dominance though only significant in right handers plus a small non-drug-using group. Right-handed patients with left-eye dominance plus left handers showed higher IMC values and less pronounced manifestation of negative symptoms. This was also true for right-eyed and right-handed cannabis users which might be due to better interhemispheric connections and less impairment of white matter structures in these patients. Thus, eye dominance plus use of cannabis should be considered in the assessment of clinical schizophrenia symptoms. Since frontostriatal dysfunction in Huntington's disease (HD) can cause deficits in theory of mind (ToM) and cognitive impairment, Eddy et al. [6] investigated the relationship between spatial perspective taking and ToM performance on the faux pas and reading the mind in the eyes (RMET) tasks plus links between ToM performance and everyday functioning in 30 patients with symptomatic HD compared with 23 controls. Additionally, ratings of quality of life, psychiatric symptoms, motor symptom severity, and functional capacity were taken for patients. They found evidence for patients with HD featuring reduced perspective taking in everyday life and poor performance on social and object-based spatial perspective taking tasks. Hence, precise cognitive and neural bases for ToM deficits in neurodegenerative states should be better specified. The relation of performance-monitoring alterations with symptom severity and dimensions in obsessivecompulsive disorder (OCD) was subject of Riesel et al.'s [7] study in 72 patients, whose symptom scores were determined via the Yale-Brown Obsessive Compulsive Scale symptom checklist, compared with 72 matched controls. Assessing electrocortical correlates of performance monitoring during a flanker task, the authors used amplitudes of the error- and correct-related negativity plus delta and theta power to quantify performance-monitoring activity. They found overactive brain processes during performance monitoring to be a common correlate of OCD independent of symptom severity and symptom dimensions, which bespeaks overactive performance monitoring to be an OCD endophenotype.

Although functional deficits in different life areas are considered as important characteristic in adult ADHD, adequate evaluation instruments to reliably and validly measure this specific variable so far were lacking. Rotger et al. [8] now tested the functioning assessment short test (FAST), previously successfully applied in other psychiatric disorders, in a case-control study with 88 adult ADHD patients and 64 controls. Analyzing feasibility, internal consistency, concurrent, and discriminant validity, plus factor analysis, the test was able to reveal significant differences in the correlation between domains with ADHD subjects featuring impairments in all areas of their life, especially regarding cognitive functioning, followed by the domains autonomy, occupational functioning, and interpersonal relationships. FAST thus is an easily applicable instrument with good psychometric properties to measure the functional level in adults with ADHD.

Investigating both selective attention and emotional bias in a large sample was aim of the study conducted by Montel et al. [9] in 51 depressed, 55 manic, and 53 euthymic bipolar disorder (BD) patients. The group compared patients' performance on the Stroop color-word (SCWT) and emotional Stroop (EST) tasks while controlling sociodemographic and clinical data. As expected from results of previous smaller studies, post hoc analysis revealed no significant differences between the single BD groups, same was true for all EST conditions-euthymic patients still treated information as did manic or depressed patients. Hence, despite their apparent clinical improvement, attentional and emotional biases in euthymic BD patients are of importance and should carefully be assessed to prevent relapses.

\section{References}

1. Fond G, Loundou A, Hamdani N, Boukouaci W, Dargel A, Oliveira J, Roger M, Tamouza R, Leboyer M, Boyer L (2014) Anxiety and depression comorbidities in irritable bowel syndrome (IBS): a systematic review and meta-analysis. Eur Arch Psychiatry Clin Neurosci. doi:10.1007/s00406-014-0502-z

2. Yen JY, Tu HP, Chen CS, Yen CF, Long CY, Ko CH (2013) The effect of serotonin 1A receptor polymorphism on the cognitive function of premenstrual dysphoric disorder. Eur Arch Psychiatry Clin Neurosci. doi:10.1007/s00406-013-0466-4

3. Ko CH, Hsieh TJ, Chen CY, Yen CF, Chen CS, Yen JY, Wang PW, Liu GC (2014) Altered brain activation during response inhibition and error processing in subjects with Internet gaming disorder: a functional magnetic imaging study. Eur Arch Psychiatry Clin Neurosci. doi:10.1007/s00406-013-0483-3

4. Woodward TS, Jung K, Smith GN, Hwang H, Barr AM, Procyshyn RM, Flynn SW, van der Gaag M, Honer WG (2013) Symptom changes in five dimensions of the positive and negative syndrome scale in refractory psychosis. Eur Arch Psychiatry Clin Neurosci. doi:10.1007/s00406-013-0460-x

5. Gorynia I, Schwaiger M, Heinz A (2014) Effects of eye dominance (left vs. right) and cannabis use on intermanual coordination and negative symptoms in schizophrenia patients. Eur Arch Psychiatry Clin Neurosci. doi:10.1007/s00406-014-0503-y

6. Eddy CM, Sira Mahalingappa S, Rickards HE (2014) Putting things into perspective: the nature and impact of theory of mind impairment in Huntington's disease. Eur Arch Psychiatry Clin Neurosci. doi:10.1007/s00406-014-0498-4

7. Riesel A, Kathmann N, Endrass T (2014) Overactive performance monitoring in obsessive-compulsive disorder is independent of symptom expression. Eur Arch Psychiatry Clin Neurosci. doi:10.1007/s00406-014-0499-3 
8. Rotger S, Richarte V, Nogueira M, Corrales M, Bosch R, Vidal R, Marfil L, Valero S, Vieta E, Goikolea JM, Torres I, Rosa A, Mur M, Casas M, Ramos-Quiroga JA (2014) Functioning assessment short test (FAST): validity and reliability in adults with attention-deficit/hyperactivity disorder. Eur Arch Psychiatry Clin Neurosci. doi:10.1007/s00406-014-0501-0

9. Montel S, Scott J, Dubois M, M'bailara K, Henry C (2013) Attentional deficits and emotional bias in bipolar disorders: comparison at different stages of the disease. Eur Arch Psychiatry Clin Neurosci. doi:10.1007/s00406-013-0477-1

10. Yen JY, Tu HP, Chen CS, Yen CF, Long CY, Ko CH (2013) Erratum to: the effect of serotonin 1A receptor polymorphism on the cognitive function of premenstrual dysphoric disorder. Eur Arch Psychiatry Clin Neurosci. doi:10.1007/s00406-013-0471-7 